\title{
Do prebiotics reduce the number of fever episodes in healthy children in their first year of life: a randomised controlled trial
}

\author{
Margriet van Stuijvenberg*, Annemieke M. Eisses, Christoph Grüber, Fabio Mosca, Sertac Arslanoglu, \\ Gaetano Chirico, Christian P. Braegger, Josef Riedler, Günther Boehm, Pieter J. J. Sauer, for the \\ Multicenter Infection Prevention Study-1 (MIPS-1) Study Group \\ Beatrix Children's Hospital, UMC Groningen, CA51, PO Box 30001, 9700 RB Groningen, The Netherlands
}

(Received 1 December 2010 - Revised 14 April 2011 - Accepted 5 June 2011 - First published online 4 October 2011)

\begin{abstract}
The objective of the present study was to assess the effect of adding specific prebiotics to standard formula feeding on the number of fever episodes in the first year of life. In the present randomised, double-blind, placebo-controlled trial in seven centres in five West European countries, 830 healthy term infants, without a first-degree family history of allergic disease, of mothers who indicated to give only formula feeding were randomised either to receive a standard non-hydrolysed cows' milk-based formula to which a mixture of specific oligosaccharides was added (prebiotics group (PG)), or to receive a similar formula without oligosaccharides (control group (CG)). A separate reference group consisted of 300 breast-fed infants. The primary outcome was the number of fever episodes prospectively documented by the parents. There was no difference in the number of fever episodes between the PG (median value 1.19; 25 th-75th percentile $0 \cdot 09-2 \cdot 34)$ and CG $(1 \cdot 16 ; 25$ th-75th percentile $0 \cdot 06-2 \cdot 38)$. The median number of fever episodes in the separate breast-feeding reference group was 1.24 (25th-75th percentile $0.51-3.45)$. There was no effect of adding specific prebiotics to standard formula feeding in reducing the number of fever episodes in the present study.
\end{abstract}

Key words: Infection: Infants: Oligosaccharides

Breast-feeding is universally recommended as the optimal feeding for infants in the first 6 months of life $e^{(1,2)}$. There is clear evidence for health benefits for both mother and child. Special attention has been given to the effect regarding infections in young children ${ }^{(3-5)}$. The protective effect of breastfeeding against infectious diseases is assumed to be provided by specific (antibody-dependent) and non-specific factors such as oligosaccharides, proteins, glycoproteins and fats. Many efforts have been made to make the composition of formula (e.g. proteins and fats) as close as possible to breast milk ${ }^{(6,7)}$.

Human milk oligosaccharides, after lactose and lipids, represent the third largest solid component in breast milk, and contribute to immune modulation and infection prevention ${ }^{(8,9)}$. They exert prebiotic function by acting as soluble analogues to epithelial receptors for specific microbes, and this prevents their adhesion to the intestinal wall ${ }^{(10,11)}$. Further, they enhance mucosal barrier function with a direct effect on intestinal maturation and trophic effects on the intestinal mucosa through their fermentation products ${ }^{(12,13)}$; lower the $\mathrm{pH}$ in the colon through the fermentation products ${ }^{(10,14)}$; and interact directly with the cells of the immune system ${ }^{(15)}$.
Thus, breast milk oligosaccharides are powerful candidates to be key components of human milk contributing to its protective effect against infections in infants. Clinical data about the effect of oligosaccharide supplementation to formula feeding on infections in young healthy children are scarce. Recently, two studies have been published pointing to a reduction of infections in the early life of infants who had an increased risk of allergic diseases ${ }^{(16-18)}$. There is no well-designed study available about its effect in low-allergyrisk populations. The aim of the present randomised, double-blind, placebo-controlled, multicentre study was to evaluate the effect of adding a mixture of specific prebiotics to standard formula feeding on the number of fever episodes in the first year of life of healthy term children.

\section{Patients and methods}

The present study (Multicenter Infection Prevention Study) was a randomised, double-blind, placebo-controlled, multicentre trial. Participating study centres were the Beatrix Children's Hospital in Groningen, The Netherlands ( $n$ 207); the Schwarzach Hospital in Schwarzach, Austria ( $n$ 181);

Abbreviations: BG, breast-feeding group; CG, control group; FAS, full-analysis set; HR, hazard ratio; PG, prebiotics group; PPS, per-protocol set. 
the University Children's Hospital in Zürich, Switzerland ( $n$ 163); the Mangiagalli Hospital in Milan, Italy ( $n$ 155); the Macedonio Melloni Hospital in Milan, Italy ( $n$ 145); the Charité-Universätsmedizin Berlin, in Berlin, Germany ( $n$ 143); the Brescia Hospital in Brescia, Italy ( $n$ 136). Parents of healthy term infants were invited with their child to participate between July 2005 and December 2007; follow-up ended in December 2008. Inclusion took place before the postnatal age of 8 weeks. Parents with babies in their first 8 weeks of life from the region of the participating centres were informed about the study in the perinatal centre (Germany), the paediatric outpatient clinic (Italy, Austria, Switzerland), or in the Well Baby Clinic (The Netherlands). Parents were asked to call back only if they were interested in the study.

Healthy term infants (gestational age 37-42 weeks) with a birth weight above the 10th percentile for gestational age according to locally applicable growth charts were included. Infants at risk for atopic diseases (having a first-degree relative to a positive history of asthma, allergic rhinitis or atopic dermatitis) were excluded ${ }^{(19)}$. Infants with metabolic disorders requiring a special diet other than standard non-hydrolysed feeding were excluded. Infants with neonatal diseases requiring antibiotic treatment, congenital diseases or malformations influencing the gastrointestinal system, and infants who had used formulas containing prebiotics or probiotics were excluded. Infants from mothers with known colonisation with group B streptococci during pregnancy, which often is an indication for antibiotic treatment, were also excluded. Infants from mothers who were suffering from HIV or hepatitis $\mathrm{B}$ were also excluded from the study. In Europe, mothers are not routinely tested for cytomegalovirus and hepatitis $\mathrm{C}$ as the prevalence of both infections in Europe is quite low. Thus, these were not considered as exclusion criteria. The study was approved by all local Ethical Review Boards of the participating centres, and written informed consent was obtained from each parent. The present study was registered with the German Clinical Trials Register DRKS00000201.

Mothers were encouraged to breast feed their infants for at least 4 months and preferably 6 months. To comply with the ethical standards not to influence breast-feeding mothers in any way to use an instant formula instead, mothers were asked their consent to participate in the study and to be randomised to one of the two formula groups only if they could not or did not fully breast feed their infants, and who received at least one bottle of formula before. Only infants of mothers who indicated before the 8th week after birth to give only formula feeding at least during the first 4 months of age were randomised. After randomisation, breast-feeding could partly be continued; after randomisation, the only substitute or supplement in case of insufficient breast-feeding was the randomised study formula. Infants of mothers who indicated to give full breast-feeding were included in the non-randomised breast-feeding reference group. In case of insufficient breast-feeding, supplemental formula feeding could be given according to the local standards at the parents' preference. If mothers could not provide enough breastfeeding any longer, the standard formula available on the local market was offered.
Randomisation to one of the two formula groups was performed immediately after study entry. Randomisation was performed stratified according to study centre. Time-balanced randomisation was performed with the software RANCODE (Professional 3.6; idv Data Analysis and Planning, Gauting, Germany), with a random permuted block size of 4 .

\section{Masking of treatment allocation}

Only the hospital pharmacist had a copy of the randomisation list with the actual treatment allocation. The parents, the study physicians and the study nurses were unaware of the group allocation. The tins and the milk powder looked and smelled identical. Infants randomised to the prebiotics group (PG) received a standard non-hydrolysed cows' milk-based formula to which a mixture of specific neutral short-chain galactooligosaccharides and long-chain fructo-oligosaccharides (ratio 9:1, Immunofortis ${ }^{\circledR} ;$ Nutricia Cuijk BV, Cuijk, The Netherlands) and specific pectin-derived acidic oligosaccharides was added. The total amount of oligosaccharides was $8 \mathrm{~g} / \mathrm{l}$, with $6 \cdot 8 \mathrm{~g} / 1$ neutral and $1.2 \mathrm{~g} / 1$ acidic oligosaccharides. Infants randomised to the control group (CG) received a similar standard non-hydrolysed cows' milk-based formula without added oligosaccharides. For both groups, starter formula was provided during the first 6 months of life, thereafter follow-on formula was offered. Infants randomised to the PG continued to receive the oligosaccharides in the follow-on formula. It was advised not to start weaning from either exclusive breast-feeding or formula feeding before the age of 4 months.

\section{Outcome measure}

The occurrence of a fever episode was the primary outcome. The observation period was until the age of 1 year. A diary provided to the parents at inclusion and filled out by parents or carers was used to ascertain the number and duration of fever episodes. Parents were asked to fill in the form every day their child had fever. They were asked to report next to fever other symptoms as well. They were instructed to take their child's temperature only if they thought the child might have fever; no further instruction was given in order not to influence normal parental care. We considered this "normal daily practice at home' the best condition for performing the study. We assumed that variations from family to family about the threshold for taking the temperature would be equally distributed between the PG and CG by randomisation (but not in the non-randomised breast-feeding group (BG)). Fever was defined as a peak rectal temperature $\geq 38.5^{\circ} \mathrm{C}$; a peak rectal temperature was the highest measured rectal temperature of at least three measurements during a $24 \mathrm{~h}$ period. We calculated a new fever episode if the time without any symptoms between febrile illnesses was more than 1 day $(24 \mathrm{~h})$. Every 2 weeks (after the age of 16 weeks every 4 weeks) contact was made with the parents, either by home visits, clinic visits or phone calls. During these interviews, also other data, including the occurrence of atopic dermatitis, were collected; the results have been published separately ${ }^{(20)}$ 
Symptoms which accompanied the fever were registered based on parental reports. Based on these reports, we retrospectively made a suspected diagnosis of the cause of the fever. Gastroenteritis was defined as a fever episode accompanied by vomiting and diarrhoea, or only diarrhoea. Upper respiratory tract infection was defined as a fever episode accompanied by a runny nose with cough or wheeze. Otitis was defined as a fever episode accompanied by a painful or discharging ear. Parents were asked to report if their child had received a vaccination within $3 \mathrm{~d}$ before the fever occurred: this was considered a vaccination-related fever if no other symptoms were reported. All fever episodes were counted, irrespective of the associated symptoms or suspected cause. Symptoms were based on the reports of the parents; a doctor's diagnosis was not considered necessary. However, if reported, a doctor's diagnosis was given priority to other suspected diagnoses. Systemic antibiotic treatment during fever was also reported by the parents.

\section{Statistical analysis}

In order to detect a mean reduction of one fever episode ( 4.5 fever episodes per year in the CG compared with 3.5 in the $P G)^{(21)}$, with a standard deviation of $3 \cdot 5$, 93\% power and significance level of 0.05 in a one-sided unpaired $t$ test, 250 formula infants were needed per group. With a $50 \%$ total dropout rate (expected 35\% mixed feeding and 15\% dropout for another reason), 500 infants per group had to be enrolled. After some time, the expectations about the expected total dropout rate could be adjusted down to about $40 \%$. To stay close to the planned timeline, the recruitment was stopped at about 400 infants per group.

All statistical analyses were performed with SAS ${ }^{\circledR}$, version 9 (SAS Institute, Cary, NC, USA). The main outcome variable was analysed using one-sided testing, which was determined a priori. All the other analyses were performed using twosided testing. Significance level for all testing was 0·05. All analyses were performed with stratification per centre, unless numbers were too small.

The primary analyses were performed using the fullanalysis set (FAS), and in some cases also using the per-protocol set (PPS). The FAS consisted of all children who had been randomised to the $\mathrm{PG}$ or $\mathrm{CG}$, or had been included in the BG (non-randomised), with the exclusion of the screening dropouts (within 8 weeks after birth). The PPS consisted of all children who completed the follow-up until their first birthday (52 weeks), and had received exclusively the prebiotic formula feeding, or the control formula feeding, during the complete follow-up, or had received exclusively breast-feeding for the first 4 months of life (20 weeks).

Children who were randomised to the PG or CG, and received additional breast-feeding, were considered as 'mixed feeding' and were analysed in the FAS only, according to the allocated formula feeding group (either the PG or CG). This may eventually diminish the magnitude of the hypothesised effect of prebiotics supplementation to infant formula feeding in the FAS analysis, but not in the PPS analysis.
We collected data about the actual exclusive intake of study formula feeding (PG and CG) or BG over time in the first 16 weeks, in order to assess the 'ingested dose' of prebiotics in the study groups at a time that no additional other feeding (fruits, vegetables and bread) was given.

Dropouts were those infants who did not complete the firstyear follow-up, because of (1) change to another formula (whether or not on the preference of the parents or on doctors' advice; change to another formula in the BG indicates that the breast-feeding was stopped completely before 4 months of age); (2) intolerance to formula (occurrence of vomiting, flatulence, cramps, colics, nappy rash or diarrhoea); (3) occurrence of any disease (as a reason to withdraw the infant from the study on the preference of the parents or on doctors' advice); (4) withdrawal (dropout for unknown reason). All dropouts were analysed in the FAS.

The main outcome variable was the rate of fever episodes (adjusted) and was defined as (number of fever episodes $+0 \cdot 05) /($ duration of observational period $+0 \cdot 1)$. By adding 0.05 and $0 \cdot 1$, the rates have been ranked to be used for statistical analysis. This adjustment was done in order to prevent 'zero' in those cases without any fever episode. Furthermore, the duration of the observational period has been taken into account ${ }^{(22)}$. To present the number of fever episodes per year, this number has been multiplied by $365 \cdot 1 \mathrm{~d}$. By using this technique, the number of fever episodes per child could be taken into account. Similar calculations were done for the number of suspected causes of fever and associated symptoms. This is the reason why those 'adjusted numbers' cannot be added up to calculate the total median adjusted number of all fever episodes.

Differences in the rate of fever episodes and number of fever episodes per year were analysed using the nonparametric van Elteren test for all centres, or the Wilcoxon rank-sum test for individual centres ${ }^{(23)}$. The analyses were performed for the first year of life (from inclusion in the study until the age of 12 months) and in the first half year (from inclusion in the study until the age of 6 months), both in the FAS and PPS datasets.

We only tested for any differences in baseline characteristics between the PG and CG (stratified regression analysis), and not the BG due to its non-randomised character. Time to first occurrence of a fever episode and recurrent fever episodes were analysed using stratified Cox proportional hazard regression analysis ${ }^{(24)}$. The time to first occurrence of a fever episode was defined as the time from inclusion to the day before the first occurrence of a fever episode. If no fever episode occurred during the observational period, the time to first occurrence equalled the duration of the observational period. The end of the observational period was either the end of the study at the age of 12 months or early dropout from the study for whatever reason. By analysing the data using this technique, any time-dependent fluctuation of the risk of having a fever episode is taken into account. Cox regression analysis was also used to examine the occurrence of repeated fever episodes. The counting process model was used, which assumes that each fever episode is independent of the other, and that a subject is at risk for a subsequent 
event, even if he had not previously experienced an event, provided he is still under observation. Since multiple events were recorded for each subject, the model also accounted for correlated observations within subjects. To model the occurrence of repeated events, all baseline variables (presented in Table 1) were used. A multivariate regression model was identified including the number of fever episodes as the dependent variable. A forward stepwise selection was conducted, with a $P$ value entry criterion of $0 \cdot 10$ and a stay criterion of $0 \cdot 10$. Due to the non-randomised character, the BG was not included in this analysis.

\section{Results}

From July 2005 to December 2007, 414 infants were randomised to the PG and 416 infants to the CG, and 300 infants were included in the BG which is the non-randomised reference group (Fig. 1). There was no statistical significant difference in dropout numbers in the PG $v$. CG $\left(\chi^{2}\right.$ test, $P=0 \cdot 23$ )

The baseline characteristics are given in Table 1 . There were no significant differences between the PG and CG. There were quite some baseline differences between the CG and BG, as can be expected due to the non-randomised character of this group (not statistically tested).
Table 2 presents the intake of feeding per study group. The exclusive intake of formula feeding showed a $10 \%$ increase over time in the first 16 weeks from about $70 \%$ up to $80 \%$ in both formula groups. The BG showed a $4 \%$ decrease in the exclusive intake of breast-feeding from about 88 to $84 \%$ over time in the first 16 weeks.

Table 3 presents the overall adjusted number of fever episodes during the first year of life. There was no significant difference between the PG and CG (median values 1.19 $v$. $1 \cdot 16, P>0 \cdot 05)$. When only those infants who completed the study were analysed (PPS), a small but significant difference in the number of fever episodes was found in the first 6 months in the PG $v$. CG: (median values $0 \cdot 13$ (25th-75th percentile $0 \cdot 12-2 \cdot 31) \quad v \cdot 0 \cdot 13(25$ th -75 th percentile $0 \cdot 12-$ 2.36), $P<0 \cdot 05$ ).

Table 4 presents the adjusted number of fever episodes during the first year of life for all infants for each participating centre separately. On a centre level, there were no significant differences between the PG $v$. CG. There was a significant difference in the adjusted number of fever episodes in the first year of life in the CG $v$. BG in the centres University Hospital Charité, Berlin, Germany (1.22 v. 2.36, P<0.05) and Zürich Children's Hospital, Zürich, Switzerland (1.26 v. 3.45, $P<0 \cdot 05)$.

The hazard ratio (HR) of having a first fever episode was 1.07 (95\% CI $0.90,1.26)$ in the PG $v$. CG. Fig. 2 presents the

Table 1. Baseline characteristics

(Median values, 25th-75th percentiles, number of subjects and percentages)

\begin{tabular}{|c|c|c|c|c|c|c|c|c|c|c|c|c|}
\hline & \multicolumn{4}{|c|}{$\mathrm{PG}(n 414)$} & \multicolumn{4}{|c|}{$\mathrm{CG}(n 416)$} & \multicolumn{4}{|c|}{$\mathrm{BG}(n 300)$} \\
\hline & Median & $\begin{array}{l}25 \text { th }-75 \text { th } \\
\text { percentile }\end{array}$ & $n$ & $\%$ & Median & $\begin{array}{l}25 \text { th }-75 \text { th } \\
\text { percentile }\end{array}$ & $n$ & $\%$ & Median & $\begin{array}{l}25 \text { th }-75 \text { th } \\
\text { percentile }\end{array}$ & $n$ & $\%$ \\
\hline Age at inclusion $(d)^{*}$ & 30 & $20-42$ & & & 32 & $21-45$ & & & 50 & $36-54$ & & \\
\hline Birth weight $(\mathrm{g})$ & 3277 & $2965-3610$ & & & 3320 & $2930-3625$ & & & 3438 & $3110-3678$ & & \\
\hline Gestational age (completed weeks) & 39 & $38-40$ & & & 39 & $38-40$ & & & 40 & $39-40$ & & \\
\hline Boys & & & 220 & 53 & & & 200 & 48 & & & 134 & 45 \\
\hline White race & & & 378 & 91 & & & 392 & 94 & & & 287 & 96 \\
\hline Vaginal delivery & & & 261 & 63 & & & 272 & 65 & & & 247 & 82 \\
\hline $\begin{array}{l}\text { Rooms in household (not } \\
\text { kitchen/bathroom) }\end{array}$ & 3 & $3-4$ & & & 3 & $3-4$ & & & 4 & $3-5$ & & \\
\hline $\begin{array}{c}\text { Number of children in } \\
\text { household }\end{array}$ & 2 & $1-2$ & & & 2 & $1-2$ & & & 2 & $1-2$ & & \\
\hline Single-parent family & & & 25 & 6 & & & 23 & 6 & & & 17 & 6 \\
\hline Furry pets in household & & & 133 & 32 & & & 150 & 36 & & & 95 & 32 \\
\hline Smoking mother & & & 79 & 19 & & & 81 & 19 & & & 21 & 7 \\
\hline Smoking father & & & 142 & 35 & & & 163 & 40 & & & 81 & 27 \\
\hline \multicolumn{13}{|l|}{ Education level of the mother } \\
\hline Primary and secondary school & & & 206 & 50 & & & 208 & 50 & & & 115 & 38 \\
\hline $\begin{array}{c}\text { Some university education/some } \\
\text { post-secondary education/ } \\
\text { technical or trade qualification }\end{array}$ & & & 138 & 33 & & & 119 & 29 & & & 107 & 36 \\
\hline Completed university degree & & & 69 & 17 & & & 89 & 21 & & & 78 & 26 \\
\hline Mother employed & & & 252 & 61 & & & 269 & 65 & & & 207 & 69 \\
\hline \multicolumn{13}{|l|}{ Education level of the father } \\
\hline Primary and secondary school & & & 184 & 46 & & & 188 & 46 & & & 101 & 34 \\
\hline $\begin{array}{c}\text { Some university education/some } \\
\text { post-secondary education/ } \\
\text { technical or trade qualification }\end{array}$ & & & 142 & 35 & & & 127 & 31 & & & 113 & 38 \\
\hline Completed university degree & & & 77 & 19 & & & 95 & 23 & & & 85 & 28 \\
\hline Father employed & & & 369 & 92 & & & 377 & 92 & & & 283 & 95 \\
\hline
\end{tabular}

PG, prebiotics group; CG, control group; BG, breast-feeding group

* Age at inclusion is similar to age at start of the study formula (in the PG and CG). 


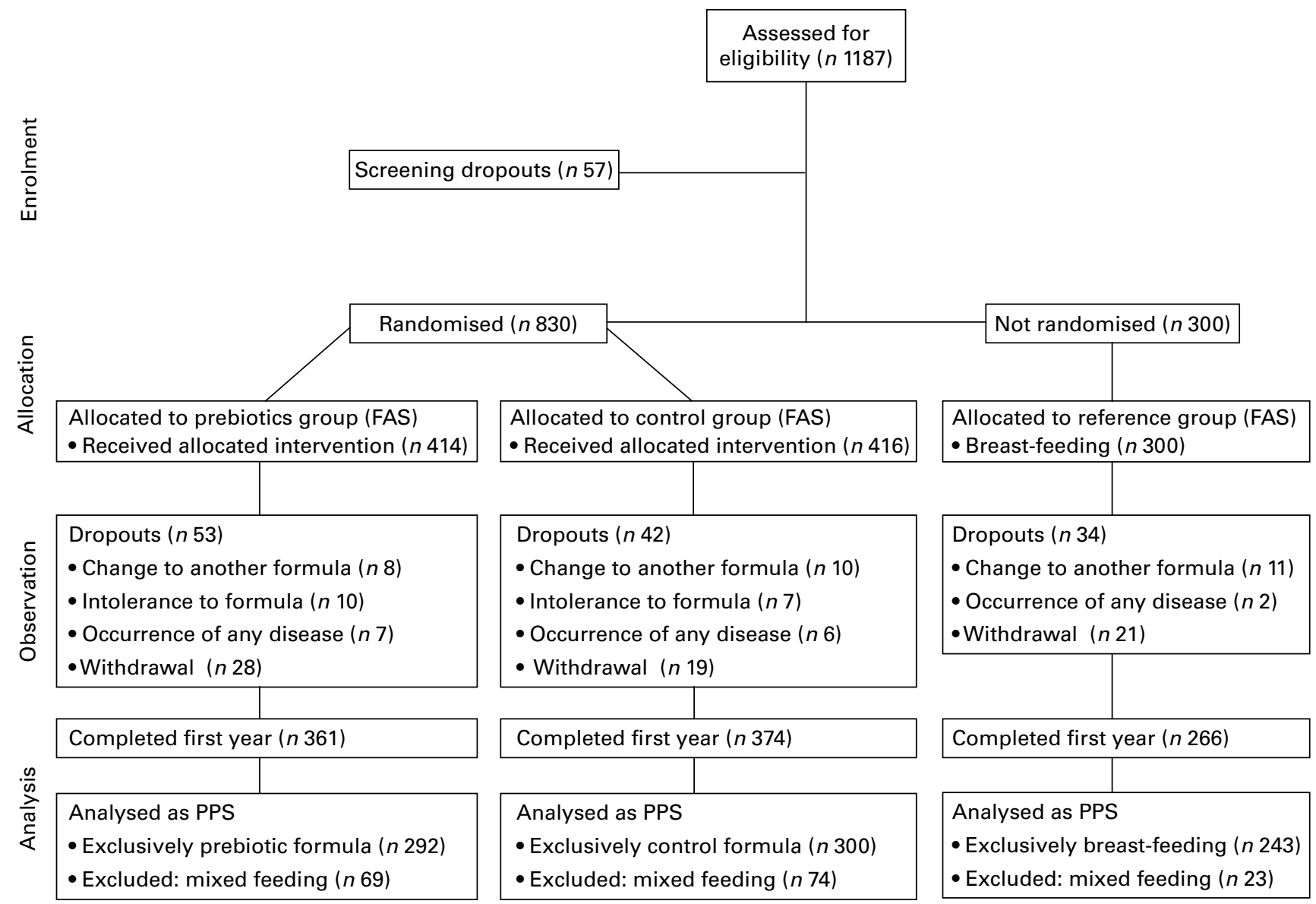

Fig. 1. Flow chart of infants participating in the study. FAS, full-analysis set; PPS, per-protocol set.

Kaplan-Meier curve of the analysis of time to first occurrence of a fever episode. The Kaplan-Meier analysis showed a probability of 0.66 of having a first fever episode after inclusion in the PG, 0.65 in the CG and 0.71 in the BG. Median survival times were 210, 220 and 208 d, respectively.

Table 5 presents the final results of the multivariate recurrent event analysis. Study group was not a significant variable influencing the rate of fever episodes. The rate of fever episodes was significantly increased by the variables: male sex (HR 1.25) and white race (HR 1.42). The rate of fever episodes was decreased by the presence of furry pets in the household (HR 0.80).

There was no difference in day-care use (more than six children in a group) during the complete follow-up between the PG and CG (repeated-measures logistic regression analysis, $P=0 \cdot 26$ ). At the time point of 20 weeks, in the PG $2.2 \%$ of the children used day care; in the CG $2.9 \%$; and in the BG 3.2\%; and at 52 weeks, these numbers were 15, 14 and $17 \%$, respectively.

The total number of fever episodes was 1697, comprising the following categories of suspected causes of fever and associated symptoms: upper respiratory tract symptoms (465; $27 \%$ ), no symptoms except fever (377; $22 \%$ ), other symptoms (298; 18\%), gastroenteritis symptoms (187; 11\%), ear infection (119; 7\%), exanthema (117; 7\%), vaccination-related fever (56; 3\%), teething (31; $2 \%)$, pneumonia/bronchiolitis (19; 1\%), urinary tract infection (15; 1\%), eye infection (12;0.5\%) and osteomyelitis (1; $0.1 \%)$. The adjusted number of the suspected causes of fever and associated symptoms in the first year of life are presented in Table 6. There were no statistical significant differences between the PG and CG.

The median adjusted numbers of fever episodes for which systemic antibiotics have been used during the first year of life were 0.05 (25th-75th percentile $0.05-0.11$ ) in the PG, 0.05 (25th-75th percentile 0.05-0.16) in the CG, and 0.05 (25th-75th percentile 0.05-0.09) in the BG. There was no statistically significant difference between the PG and CG.

There were no serious adverse events related to the intervention. Details will be published separately.

Table 2. Exclusive study formula and exclusive breast-feeding per 2-week time point in the first 16 weeks of life

(Number of infants and percentages)

\begin{tabular}{|c|c|c|c|c|c|c|}
\hline & \multicolumn{2}{|c|}{$\mathrm{PG}(n$ 414) } & \multicolumn{2}{|c|}{$\mathrm{CG}(n 416)$} & \multicolumn{2}{|c|}{$\mathrm{BG}(n 300)$} \\
\hline & $n$ & $\%$ & $n$ & $\%$ & $n$ & $\%$ \\
\hline 8 weeks & 286 & $69 \cdot 1$ & 284 & 68.3 & 264 & 88.0 \\
\hline 10 weeks & 301 & $72 \cdot 7$ & 309 & 74.3 & 264 & 88.0 \\
\hline 12 weeks & 325 & 78.5 & 332 & 79.8 & 264 & 88.0 \\
\hline 14 weeks & 321 & $77 \cdot 5$ & 333 & $80 \cdot 0$ & 256 & $85 \cdot 3$ \\
\hline 16 weeks & 340 & 82.1 & 343 & 82.5 & 253 & $84 \cdot 3$ \\
\hline
\end{tabular}

PG, prebiotics group; $C G$, control group; $B G$, breast-feeding group. 
Table 3. Adjusted number of fever episodes per year

(Median values, 25th-75th percentiles and number of infants)

\begin{tabular}{|c|c|c|c|c|c|c|}
\hline & \multicolumn{2}{|r|}{$P G$} & \multicolumn{2}{|r|}{ CG } & \multicolumn{2}{|r|}{$B G$} \\
\hline & Median & 25th-75th percentile & Median & 25th-75th percentile & Median & 25th-75th percentile \\
\hline \multicolumn{7}{|l|}{ FAS } \\
\hline$n$ & & 414 & & 416 & & 300 \\
\hline \multicolumn{7}{|l|}{ Follow-up time } \\
\hline Inclusion - 12 months of age & $1 \cdot 19$ & $0.09-2.34$ & $1 \cdot 16$ & $0.06-2.38$ & $1 \cdot 24$ & $0.51-3.45$ \\
\hline Inclusion - 6 months of age & 0.13 & $0 \cdot 12-2 \cdot 31$ & 0.13 & $0.12-2 \cdot 36$ & 0.14 & $0.13-2.63$ \\
\hline \multicolumn{7}{|l|}{ PPS } \\
\hline$n$ & & 292 & & 300 & & 243 \\
\hline \multicolumn{7}{|l|}{ Follow-up time } \\
\hline Inclusion - 12 months of age & $1 \cdot 19$ & $0.09-2.34$ & $1 \cdot 16$ & $0.06-2.38$ & 1.24 & $0.51-3.45$ \\
\hline Inclusion - 6 months of age & 0.13 & $0 \cdot 12-2 \cdot 31$ & $0 \cdot 13^{*}$ & $0 \cdot 12-2 \cdot 36$ & $0 \cdot 14$ & $0.13-2 \cdot 63$ \\
\hline
\end{tabular}

PG, prebiotics group; CG, control group; BG, breast-feeding group; FAS, full-analysis set; PPS, per-protocol set.

* Median value was significantly different from that of the $P G(P<0.05)$.

\section{Discussion}

In the present study of healthy term infants, we found no significant difference in the number of fever episodes between the PG and CG in the first year of life.

As suspected causes of fever, mainly respiratory tract infections and gastroenteritis were counted. The frequency of the suspected causes of fever and associated symptoms was more or less equally distributed as in other relevant studies $^{(17,18,21)}$. However, we did not find any differences in the suspected causes of fever between the PG and CG.

Results of previous studies point to favour children receiving formula supplied with prebiotics. A randomised, placebo-controlled, single-centre study of a prebioticsupplemented ( $8 \mathrm{~g} / 1$ galacto- and fructo-oligosaccharides) formula showed a significant reduction in the number of infectious episodes documented by a paediatrician in the first 6 months in the $\mathrm{PG}^{(16)}$. In the follow-up of this cohort, in addition, the occurrence of fever episodes of $\geq 38.5^{\circ} \mathrm{C}$ reported by the parents was significantly reduced in the $\mathrm{PG}^{(17)}$. A Finnish randomised, placebo-controlled trial of 'synbiotic' treatment of four probiotic species (mothers) and the same probiotics in addition to $0.8 \mathrm{~g} / 1$ galacto-oligosaccharides (their infants) showed a reduction of respiratory infections in the 'synbiotic' treatment group. In contrast to the present results, both studies showed a reduction in the use of antibiotic treatment in the prebiotic and 'synbiotic' groups, respectively ${ }^{(17,18)}$.

From the most recent multicentre, open-label, randomised controlled trial, it is difficult to draw any conclusions because of the open design and presentation of per-protocol analysis results only ${ }^{(25)}$. In summary, unlike the present study, these previous studies showed a reduced number of infections in infants receiving a formula supplied with prebiotics.

The difference in results may be explained by different characteristics of the studies. The previous studies mainly counted physician-diagnosed infections. However, diagnosis may not play an important role since the incidence of fever episodes and infections is low. Previous studies included infants with an increased risk for atopy, probably prone to respiratory infections. Both previous studies ${ }^{(17,18)}$ showed a decrease in respiratory infections at 2 years of age indeed. The present Multicenter Infection Prevention Study population is a healthy population: all children were identified by preventive health care visits, and had a low risk for allergy. Based on the very low rate of fever episodes in the present study, we may also consider the study underpowered to find an effect of the prebiotic mixture on the number of fever episodes. However, if much more participants would be necessary to find an effect, the clinical relevance would be very small. In the additional sub-analysis of the PPS set

Table 4. Adjusted number of fever episodes per year per study centre (Median values, 25th-75th percentiles and number of infants)

\begin{tabular}{|c|c|c|c|c|c|c|c|}
\hline & \multirow[b]{2}{*}{$n$} & \multicolumn{2}{|c|}{$\mathrm{PG}(n 414)$} & \multicolumn{2}{|c|}{ CG (n 416) } & \multicolumn{2}{|c|}{$\mathrm{BG}(n 300)$} \\
\hline & & Median & $\begin{array}{l}\text { 25th-75th } \\
\text { percentile }\end{array}$ & Median & $\begin{array}{l}\text { 25th-75th } \\
\text { percentile }\end{array}$ & Median & $\begin{array}{l}25 \text { th }-75 \text { th } \\
\text { percentile }\end{array}$ \\
\hline Beatrix Children's Hospital, Groningen, The Netherlands & 207 & $1 \cdot 21$ & $0.49-2 \cdot 70$ & $1 \cdot 21$ & $0.06-2.39$ & $1 \cdot 20$ & $0.06-2.23$ \\
\hline Schwarzach Hospital, Schwarzach, Austria & 181 & $1 \cdot 20$ & $1.09-2.35$ & $1 \cdot 20$ & $0.32-3.25$ & 1.24 & $0.70-3.34$ \\
\hline University Children's Hospital, Zürich, Switzerland & 163 & $1 \cdot 76$ & $0.27-3 \cdot 30$ & $1 \cdot 26$ & $0.06-3.07$ & $3 \cdot 45^{\star}$ & $1 \cdot 23-5 \cdot 01$ \\
\hline Mangiagalli Hospital, Milan, Italy & 155 & 0.53 & $0.05-2 \cdot 14$ & 1.07 & $0.05-2.18$ & 0.83 & $0.06-1 \cdot 13$ \\
\hline Macedonio Melloni Hospital, Milan, Italy & 145 & $1 \cdot 22$ & $0.06-2.28$ & $1 \cdot 13$ & $0.05-2.25$ & 3.29 & $0.13-3.92$ \\
\hline Charité Universitätsmedizin, Berlin, Germany & 143 & $2 \cdot 15$ & $1 \cdot 15-3 \cdot 22$ & 1.22 & $0.36-2.38$ & $2 \cdot 36^{\star}$ & $1.20-4.69$ \\
\hline Brescia Hospital, Brescia, Italy & 136 & 1.05 & $0.06-1.25$ & $1 \cdot 10$ & $0.06-2 \cdot 27$ & $1 \cdot 21$ & $0.06-2.39$ \\
\hline Total & 1130 & 1.19 & $0.09-2.34$ & 1.16 & $0.06-2.38$ & $1 \cdot 24^{*}$ & $0.51-3.45$ \\
\hline
\end{tabular}

PG, prebiotics group; CG, control group; BG, breast-feeding group.

* Median values were significantly different from those of the CG $(P<0.05)$. 


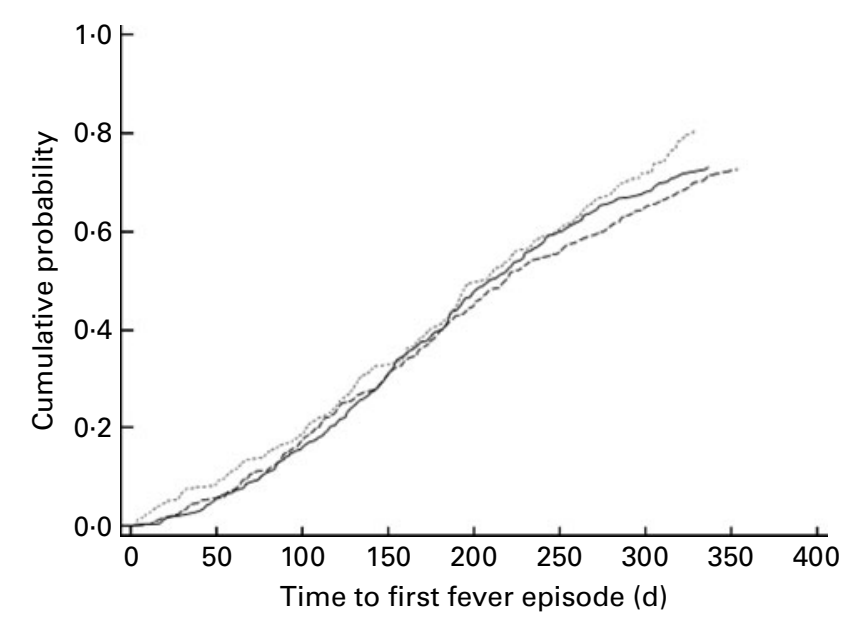

Fig. 2. Cumulative probability over time of a first fever episode by treatment group (full-analysis set). Prebiotics group (-), control group (--) and breastfeeding group (-.).

for the first 6 months of life, we found a significant but very small difference in the number of fever episodes in the first 6 months in the PG $v$. CG (both median values 0.13 ) in favour of the PG. These median values are equal, but a statistical significant difference exists, which is possible because of the non-parametric nature of the test. However, the difference, although significant, is probably not clinically relevant. The low incidence of fever episodes in the present study also plays a role. We may hypothesise about an effect only existing at the time when intake of formula is high and less diluted by other feedings. In the present study, the actual use of the study formula, and thus the magnitude of the ingested dose in the first 16 weeks, was similar in the PG and CG. Between 70 and $80 \%$ of the infants received the study formula only. A minority of $20-30 \%$ received additional breast milk. This may have influenced the study results in diluting the beneficial effect of the prebiotic mixture, if any: prebiotics have been provided to the infants randomised to the CG by this additional breast milk.

Overall, the number of fever episodes per year is much lower than we had expected previously ${ }^{(21)}$. In the study of Wald, in which type of nutrition was not accounted for, children in a home care setting showed $4 \cdot 7-7 \cdot 1$ infections in the first year of life, depending on setting of care. Upper respiratory tract infections accounted for almost $85 \%$ of all infections; and nasal discharge or nasal congestion was considered to be an infection. Severe infections, defined as fever higher than $39.0^{\circ} \mathrm{C}$, or a doctor's consult, occurred $2-3.5$ times in the first year of life in infants. These results may indicate that fever episodes are a more robust indicator of an infection compared with other symptoms in the first year of life. Fever episodes reported by the parents as an indicator of infection have been presented in a few other studies. A recent study presented a number of $0 \cdot 1-0 \cdot 4$ fever episodes per child in 12 weeks in healthy term children aged 4-10 months in daycare centres in Israel ${ }^{(26)}$. The study of Arslanoglu presented 3.9 (placebo group) $v \cdot 2 \cdot 2$ (PG) fever episodes in 2 years in children with an increased risk of atopy. In this study, it has not been documented whether fever was an accompanying symptom of infections diagnosed by a physician. A comparison with the present data cannot be made easily ${ }^{(17)}$. We may hypothesise about a decreasing occurrence of infections in the first year of life in the relatively rich parts of the world. An explanation might be the introduction of vaccinations against Haemophilus influenzae type B and Streptococcus pneumoniae, as well as improved socio-economic factors and possibly nutritional habits. In case of this situation of low numbers of fever episodes in the first year of life of healthy term children, nutritional interventions, such as prebiotic supplementation, may not have an additional substantial reducing effect. Some differences regarding fever episodes between countries existed: in Berlin, Germany, and Zürich, Switzerland, higher numbers of fever episodes in the BG were reported. We do not know why such a difference exists between countries, but reporting bias in the sense of a higher level of recognising and reporting of fever in the BG in these countries could definitely play a role.

In contrast to the hypothesised decreasing number of infections, there is strong evidence of an increase in allergic symptoms in young children ${ }^{(27)}$ (http://www.cdc.gov/asthma). Studies have suggested a role of prebiotics in the prevention of atopic dermatitis $^{(17,20,28)}$ (http://www.cdc.gov/asthma).

Regression analysis revealed factors influencing the number of fever episodes in the present study. Males had a higher number of fever episodes than females (about 25\%), white children had a higher number of fever episodes than others (about 40\%), and children in households with a furry pet had a lower number of fever episodes (about 20\%). Being a boy (compared with being a girl) was the only factor identified in another study previously ${ }^{(20)}$. To our knowledge, the presence of furry pets in the household was not identified earlier as a factor influencing (that is reducing) the number of fever episodes. Ethnicity was identified as a factor influencing the number of fever episodes in the present study. However, the prevalence of different ethnicities was very low in the present study (Table 1), so this factor should be interpreted with care.

In contrast to previous findings, the present data suggest a higher number of fever episodes in the BG. An explanation might be that parents in the BG may have a higher awareness, or a more precise way, to recognise and report fever in their child compared with parents giving formula feeding. A higher education level of parents choosing to give breastfeeding may be a part of the explanation for this 'reporting bias'. In the present study, breast-fed infants lived in relatively

Table 5. Multivariate regression analysis of recurrent event analysis final model

(Hazard ratios (HR) and $95 \%$ confidence intervals)

\begin{tabular}{lcc}
\hline & HR & $95 \% \mathrm{Cl}$ \\
\hline Boys & 1.25 & $1.08,1.43$ \\
White race & 1.42 & $1.07,1.87$ \\
Furry pets in the household & 0.80 & $0.68,0.94$ \\
Study group & & $0.86,1.14$ \\
$\quad$ Prebiotics group & 0.99 & Reference category \\
$\quad$ Control group & \multicolumn{2}{c}{ Reg } \\
\hline
\end{tabular}


Table 6. Adjusted number of suspected causes of fever and associated symptoms per year

(Median values and 25th-75th percentiles)

\begin{tabular}{|c|c|c|c|c|c|c|}
\hline \multirow[b]{2}{*}{ FAS } & \multicolumn{2}{|c|}{$\mathrm{PG}(n 414)$} & \multicolumn{2}{|c|}{$\mathrm{CG}(n 416)^{\star}$} & \multicolumn{2}{|c|}{$\mathrm{BG}(n 300)$} \\
\hline & Median & 25th-75th percentile & Median & 25th-75th percentile & Median & 25th-75th percentile \\
\hline Upper respiratory tract infection & 0.05 & $0.05-1.03$ & 0.05 & $0.05-1.03$ & 0.05 & $0.05-1.04$ \\
\hline No symptoms except fever & 0.05 & $0.05-0.33$ & 0.05 & $0.05-0.27$ & 0.05 & $0.05-1.03$ \\
\hline Other symptoms & 0.05 & $0.05-0.14$ & 0.05 & $0.05-0.16$ & 0.05 & $0.05-1.01$ \\
\hline Gastroenteritis & 0.05 & $0.05-0.09$ & 0.05 & $0.05-0.07$ & 0.05 & $0.05-0.05$ \\
\hline Ear infection & 0.05 & $0.05-0.05$ & 0.05 & $0.05-0.05$ & 0.05 & $0.05-0.05$ \\
\hline Viral exanthema & 0.05 & $0.05-0.05$ & 0.05 & $0.05-0.05$ & 0.05 & $0.05-0.05$ \\
\hline Vaccination-related fever & 0.05 & $0.05-0.05$ & 0.06 & $0.05-0.05$ & 0.06 & $0.06-0.05$ \\
\hline Teething & 0.05 & $0.05-0.05$ & 0.05 & $0.05-0.05$ & 0.05 & $0.05-0.05$ \\
\hline Pneumonia/bronchiolitist & 0.05 & $0.05-0.05$ & 0.05 & $0.05-0.05$ & 0.05 & $0.05-0.05$ \\
\hline Urinary tract infection $†$ & 0.05 & $0.05-0.05$ & 0.05 & $0.05-0.05$ & 0.05 & $0.05-0.05$ \\
\hline Eye infection & 0.05 & $0.05-0.05$ & 0.05 & $0.05-0.05$ & 0.05 & $0.05-0.05$ \\
\hline Osteomyelitis† & 0.05 & $0.05-0.05$ & 0.05 & $0.05-0.05$ & 0.05 & $0.05-0.05$ \\
\hline All fever episodes & $1 \cdot 19$ & $0.09-2.34$ & $1 \cdot 16$ & $0.06-2.38$ & $1 \cdot 24$ & $0.51-3.45$ \\
\hline
\end{tabular}

PG, prebiotics group; CG, control group; BG, breast-feeding group; FAS, full-analysis set.

* Median values were not significantly different from those of the PG (one-sided van Elteren test).

† Doctor's diagnosis only.

healthy circumstances (Table 1). In previous studies, similar differences between breast-fed and formula-fed infants were found $^{(3,4)}$. We may hypothesise that, assuming that nowadays in the relatively rich parts of the world, socio-economic (hygiene) standards are above a certain level for healthy term children in general, this surplus of healthy circumstances in breast-fed children, or the breast-feeding itself, does not protect against infections at a scale high enough to be detected anymore. This lack of effect of type of feeding on the number of fever episodes may be reassuring for mothers who for any reason are not able to provide breast milk to her child. Follow-up studies are needed to evaluate if there is an effect at an older age. Previous studies have compared the frequency of infections in breast-fed and formula-fed healthy term infants, all showing a lower number of infections in breast-fed children. Difficulties in interpreting these results arise from studies presenting a positive effect on a selection of symptoms and age categories only ${ }^{(3,5,29)}$, some studies do not report a comparison with formula feeding ${ }^{(30)}$, or do not present data about the prevalence of breast-feeding in the study population $^{(29)}$ and one study showed a large reducing effect of exclusive and prolonged breast-feeding for 6 months on hospitalisation frequency; in this study population, there was a high estimated hospitalisation frequency but a low prevalence of exclusive and prolonged breast-feeding for at least 6 months $^{(5)}$. Due to the non-randomised character of the BG in all these previous studies and the present study, it remains difficult to quantify the effect of breast-feeding on the number of infections in healthy term infants in low-risk parts of the world. Irrespective of the difficulty to show high-level evidence, as clinicians, we certainly do agree with the international guidelines to promote breast-feeding for all infants ${ }^{(1,2)}$

\section{Conclusion}

In the present study, specific prebiotics added to standard formula feeding do not decrease the number of fever episodes in the first year of life in healthy term infants. If there is an effect, it probably is very small and clinically of minor relevance.

\section{Acknowledgements}

The study has been sponsored by Danone (formerly Numico), a company that develops and sells specialised nutrition worldwide. Conflicts of interest: A. M. E. and G. B. have received financial support from Danone Research for the submitted work; C. G. has received honorarium for presentation; C. P. B., P. J. J. S. and J. R. have a membership of the advisory board with Danone Research. All authors read and approved the submission of this version of the manuscript and take full responsibility for the manuscript. M. v. S. wrote the first draft of the manuscript. She did not receive any form of payment. All authors contributed equally and substantially to the study design, data collection, data analysis and interpretation, and to the drafting of the manuscript.

The Multicenter Infection Prevention Study-1 (MIPS-1) Study Group consisted of the following members: Groningen, The Netherlands - Pieter Sauer, Margriet van Stuijvenberg, Annemieke Eisses, Jitty Streurman; Berlin, Germany Ulrich Wahn, Christoph Grüber, Juliane Wauer, Kirn Parasher, Madeleine Wust, Ingrid Lawnitzak, Marion Trentmann, Gabi Schulz, Eckard Hamelmann; Mangiagalli Hospital, Milan, Italy - Paola Roggero, Fabio Mosca, Pasca Piemontese, Maria Lorella Gianni, Anna Orsi, Valeria Puricelli; Macedonio Melloni Hospital, Milan, Italy - Sertac Arslanoglu, Guido Moro, Silvia Rizzardi, Elisa Rigotti, Laura Tandoi, Michela Coppola; Zürich, Switzerland - Christian Braegger, Michael Friedt, Rebecca Koller, Petra Martel, Sarah Nussbaumer, Daniela Rogler, Tamar Stricker; Schwarzach, Austria - Josef Riedler, Margret Macheiner, Christine Tyma; Brescia, Italy Gaetano Chirico, Gasparoni Antonella, Garzoli Elena, Offer Chiara, Spinoni Vania, Iacono Graziella, Stellini Maria; and Danone Research - Jan van der Mooren, Chryssanthi Kiriakidou, Johan Garssen, Leon Knippels, Jan Knol, Kaouther Ben Amor, Günther Boehm, Jürgen Jelinek, Bernd 
Stahl, Esther König, Anja Frings, Anja Wittke, Yvonne Zens, Stephanie Cremers, Kathrin Friedrichs, Janine Gerloff, Gemma Bruno, Ayako Fischer.

\section{References}

1. American Academy of Pediatrics. Policy Statement. Section on Breastfeeding (2005) Breastfeeding and the use of human milk. Pediatrics 115, 496-506.

2. Agostoni C, Braegger C, Decsi T, et al. (2009) Breast-feeding: a commentary by the ESPGHAN Committee on Nutrition. $J$ Pediatr Gastroenterol Nutr 49, 112-125.

3. Howie PW, Forsyth JS, Ogston SA, et al. (1990) Protective effects of breast feeding against infection. BMJ 300, 11-16.

4. Dewey KG, Heining MJ \& Nommsen-Rivers LA (1995) Differences in morbidity between breast fed and formula fed infants. Pediatrics 126, 696-702.

5. Quigley MA, Kelly YJ \& Sacker A (2007) Breastfeeding and hospitalization for diarrheal and respiratory infection in the United Kingdom Millennium Cohort Study. Pediatrics 199, e837-e842.

6. Turck D, Grillon C, Lachambre E, et al. (2006) Adequacy and safety of an infant formula with a protein/energy ratio of $1.8 \mathrm{~g} / 100 \mathrm{kcal}$ and enhanced protein efficiency for term infants during the first 4 months of life. J Pediatr Gastroenterol Nutr 43, 364-371.

7. Carnielli VP, Luijendijk IHT, Van Goudoever JB, et al. (1996) Structural position and amount of palmitic acid in infant formulas: effects on fat, fatty acid, and mineral balance. J Pediatr Gastroenterol Nutr 23, 553-560.

8. Newburg DS \& Walker WA (2007) Protection of the neonate by the innate immune system of developing gut and human milk. Pediatr Res 61, 2-8.

9. Bode L (2006) Recent advances on structure, metabolism, and function of human milk oligosaccharides. J Nutr 136, 2127-2130.

10. Forchielli ML \& Walker WA (2005) The role of gut-associated lymphoid tissues and mucosal defence. Br J Nutr 93, S41-S48.

11. Guggenbichler JP, De Bettingnies-Dutz A, Meissner P, et al. (1997) Acidic oligosaccharides from neutral sources block adherence of E. coli on uroepithelial cells. Pharm Pharmacol Lett 7, 35-38.

12. Veereman-Wauters G (2005) Application of prebiotics in infant foods. Br J Nutr 93, S57-S60.

13. Kuntz S, Rudloff S \& Kunz C (2008) Oligosaccharides from human milk influence growth-related characteristics of intestinally transformed and non-transformed intestinal cells. Br J Nutr 99, 462-471.

14. Moro G, Minoli I, Mosca M, et al. (2002) Dosage related bifidogenic effects of galacto- and fructo-oligosaccharides in formula fed term infants. J Pediatr Gastroenterol Nutr 34, 291-295.

15. Eiwegger T, Stahl B, Schmitt J, et al. (2004) Human milk derived oligosaccharides and plant derived oligosaccharides stimulate cytokine production of cord blood T-cells in vitro. Pediatr Res 56, 536-540.

16. Arslanoglu S, Moro GE \& Boehm G (2007) Early supplementation of prebiotic oligosaccharides protects formula-fed infants against infections during the first 6 months of life. J Nutr 137, 2420-2424.

17. Arslanoglu S, Moro GE, Schmitt J, et al. (2008) Early dietary intervention with a mixture of prebiotics oligosaccharides reduces the incidence of allergic manifestations and infections during the first two years of life. J Nutr 138, 1091-1095.

18. Kukkonen K, Savilahti E, Haahtela T, et al. (2008) Long-term safety and impact on infection rates of postnatal probiotic and prebiotic (synbiotic) treatment: randomized, doubleblind, placebo-controlled trial. Pediatrics 122, 8-12.

19. Hoest A, Koletzko B, Dreborg S, et al. (1999) Dietary products used in infants for treatment and prevention of food allergy. Arch Dis Child 81, 80-84.

20. Grüber C, van Stuijvenberg M, Mosca F, et al. (2010) Reduced occurrence of early atopic dermatitis because of immunoactive prebiotics among low-atopy-risk infants. J Allergy Clin Immunol 126, 791-797.

21. Wald ER, Dashefsky B, Byers C, et al. (1988) Frequency and severity of infections in day care. $J$ Pediatr 112, 540-546.

22. Moecks J, Koch GG, Scott M, et al. (2004) Measures of morbidity in clinical studies with recurrent skeletal complications. J Biopharm Stat 14, 415-437.

23. Zhao YD (2006) Sample size estimation for the van Elteren test - a stratified Wilcoxon-Mann-Whitney test. Statist Med 26, 2675-2687.

24. Hosmer DW, Lemeshow S \& May S (2008) Other models and topics. In Applied Survival Analysis: Regression Modelling of Time-to-Event Data, Chapter 9, 2nd ed., pp. 286-351. New York: Wiley-Interscience.

25. Bruzzese E, Volpicelli M, Squeglia V, et al. (2009) A formula containing galacto- and fructo-oligosaccharides prevents intestinal and extra-intestinal infections: an observational study. Clin Nutr 28, 156-161.

26. Weizman Z, Asli G \& Alsheiki A (2005) Effect of a probiotics infant formula on infections in child care centres: comparison of two probiotics agents. Pediatrics 115, 5-9.

27. Jarvis D \& Burney P (1998) ABC of allergies - the epidemiology of allergic disease. BMJ 316, 607-610.

28. Moro G, Arslanoglu S, Stahl B, et al. (2006) A mixture of prebiotics oligosaccharides reduces the incidence of atopic dermatitis during the first six months of age. Arch Dis Child 91, 814-819.

29. Kramer MS, Chalmers B, Hodnett ED, et al. (2001) Promotion of Breastfeeding Intervention Trial (PROBIT) - a randomized trial in the Republic of Belarus. JAMA 285, 413-420.

30. Chantry CJ, Howard CR \& Auinger P (2006) Full breastfeeding duration and associated decrease in respiratory tract infection in US children. Pediatrics 117, 425-432. 